\title{
Demands on surgical inpatient services after mass mammographic screening
}

\author{
LARS HOLMBERG, HANS-OLOV ADAMI, INGEMAR PERSSON, TORE LUNDSTRÖM, \\ LASZLO TABAR
}

\begin{abstract}
The changes in the demand for surgical inpatient care created by mammographic screening for breast cancer were analysed by comparing two counties, one with and one without a mass screening campaign. A comprehensive computerised register of inpatient care in the region was used. The results indicate that population based screening offered to women above 40 years and repeated every two to three years will increase the number of operations required for breast cancer and inpatient days by at least $150 \%$ during the initial screening round. During the second round the figures tend to return to previous levels. Of decisive importance for the demands on health service resources are the specificity of screening, the duration of the first screening round, and the age groups included.
\end{abstract}

\section{Introduction}

The age standardised mortality from cancer of the breast has remained stable or has slowly increased throughout Sweden and the rest of the world for several decades. ${ }^{12}$ The way to improvement seems to lie in identifying and eliminating causal factors. Such factors are most probably related to lifestyle and environment, ${ }^{3}$ but their exact nature is as yet largely unknown.

Much hope has, therefore, been placed on two possible ways of increasing the rate of cure-adjuvant systemic treatment with cytotoxic or endocrine agents and screening procedures aimed at diagnosis before systemic dissemination of the disease has occurred.

Falu lasarett, Falun, Sweden

LARS HOLMBERG, MD, senior registrar, department of surgery

LASZLO TABAR, MD, associate professor, department of mammography

University Hospital, Uppsala, Sweden

HANS-OLOV ADAMI, MD, associate professor, department of surgery

INGEMAR PERSSON, MD, senior registrar, department of obstetrics and gynaecology

Uppsala Data Center, Uppsala, Sweden

TORE LUNDSTRÖM, BSC, programmer

Correspondence to: Dr Lars Holmberg.
Although adjuvant systemic treatment has recently been proved to prolong survival in large subsets of patients, ${ }^{4}$ the size of its effect has generally been limited. Moreover, chemotherapy, in particular, has definite disadvantages related to short term toxicity and morbidity, possible long term side effects, psychological consequences, and costs.

Against this background, the fact that mammographic screening has achieved highly significant reductions in mortality from breast cancer ${ }^{5-8}$ seems to offer the hope of major progress in cancer control. In a Swedish trial the reduction in mortality from breast cancer within five to seven years approached $40 \%$ among women older than 50 years. ${ }^{8}$ These results presented a clear challenge to the health authorities in Sweden (the county councils) to consider implementing population based screening.

As such screening demands resources, rational planning of surgical care is one of the prerequisites for a cost effective design.

\section{Methods}

To assess the planning requirements we used a unique computerised register, maintained by the National Board of Health and Welfare, which documents all admissions for inpatient care to the hospitals of the Uppsala health service region. This region includes six counties, one of which had participated in a screening programme. ${ }^{8}$ Mammographic screening has been carried out in Kopparberg County since 1977. Comparisons for 1974-83 were made with Uppsala County, where there was no mass screening. An analysis of the time related changes in numbers of surgical procedures and other demands on health service resources after mass mammographic screening could therefore be based on data from the register.

\section{COMPUTERISED REGISTER OF HOSPITAL INPATIENT CARE}

The computerised register of inpatient care was begun in 1964 to help in the planning of health care and with medical research. ${ }^{9}$ The total population of the Uppsala health service region is about $11 / 2$ million (about $16 \%$ of the total Swedish population). The information entered in each case includes the national identity number given to all residents in Sweden, domicile, dates of admission and discharge, clinic, diagnosis, surgical operations, and so on. The diagnoses are codified according to the International Classification of Diseases (8th revision) and are checked and supplemented as necessary. An analysis in 1965 showed that only $0.4 \%$ of all patients discharged from the region's hospitals had not been included in the register. ${ }^{10}$ 
In 1978 the population of Kopparberg County was 284490 , including 114813 women aged 15 or more. The corresponding figures for Uppsala County were 241722 and 96929 .

\section{INCLUSION CRITERIA}

The subjects in the present investigation comprised all those in the register who $(a)$ were women aged 15 years or more; $(b)$ lived in Uppsala or Kopparberg County; $(c)$ were admitted in 1974-83 to any of the three departments of surgery in Uppsala County or the four departments of surgery in Kopparberg County with (d) a diagnosis (ICD 8) coded as 174 (malignant mammary tumour) or $217,233,610,611.01,611.02$, or 611.91 (benign mammary lesion).

\section{SURGICAL CARE}

All diagnoses of breast cancer were based on histological or cytological examination. Uniform principles of treatment were used throughout, except in 1975-7, when women with stage II disease in Kopparberg County were given adjuvant cyclophosphamide, methotrexate, and fluorouracil for a

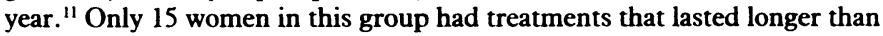
December 1977. Patients given cyclophosphamide, methotrexate, and fluorouracil were admitted to hospital for one day of each treatment course, which influenced the figures for hospital stay. During the screening period in Kopparberg patients were treated according to the same principles, irrespective of whether the tumour was detected at screening or not.

Operations for diagnosis and treatment were coded for computer storage. Biopsy and mastectomy performed in a single session were coded as two operations. All patients were thoroughly investigated before surgery (undergoing mammography and cytology) to minimise the use of frozen section immediately before mastectomy. After 1982 frozen section was rarely used, but techniques to preserve the breast were introduced for a selected group of women with stage I tumours. The two stage procedures sometimes also required for such surgery did not appreciably influence the overall figures for operations per patient. The mean number of coded operations per surgically treated patient in 1974-83 averaged 1.14 (SE 0.04) in Kopparberg County and $1 \cdot 18(0 \cdot 08)$ in Uppsala.

In Uppsala County women with recurrent breast cancer are treated at the University Hospital's department of oncology. As there is no oncology clinic in Kopparberg County patients with recurrences of breast cancer are treated at the surgical clinics. There are no private facilities for inpatient care of breast cancer in the region.

\section{SCREENING TRIAL IN KOPPARBERG COUNTY}

A screening trial with single view mammography as the only means of primary detection has been under way in Kopparberg County since October 1977. The study is part of the Swedish population based, prospective, randomised trial in Ostergötland and Kopparberg County, and its details and early results have been reported. ${ }^{8}{ }^{12}$ In Kopparberg two thirds (47 389) of all women aged 40 or more were randomised to be offered screening, and the remaining third (22658) were randomly allocated to a control group. The first round of screening, "prevalence screening," was completed in May 1980 , and the second round was completed in June 1983. Women aged 40-49 at entry to the trial were then invited for repeat examination every 24 months and older women every 34 months. Among those invited, $89 \%$ of those aged under 75 responded. The participation rate among older women fell to about $50 \%$.

The sensitivity achieved in the first round of screening was $95 \cdot 5 \%$, and the specificity was $\mathbf{9 8} \cdot 9 \% .^{13}$

\section{Results}

\section{OVERALL HEALTH CARE CONSUMPTION}

Figure 1 shows the mean and the median duration of hospital stay for women with breast cancer. In Uppsala County the mean (SE) duration fell between 1970 and 1974 (from $15 \cdot 0(2 \cdot 2)$ to $11 \cdot 1(1 \cdot 1)$ days), coinciding with a shift from radical to modified radical mastectomy. Afterwards, the annual figures remained fairly stable and within the limits of random variation until 1984. The figures for median duration of each hospital stay closely paralleled this general pattern (fig 1).

The corresponding figures for Kopparberg County were strongly influenced in 1975-7 by the adjuvant chemotherapy programme with repeated one day stays in hospital before the start of the screening programme in

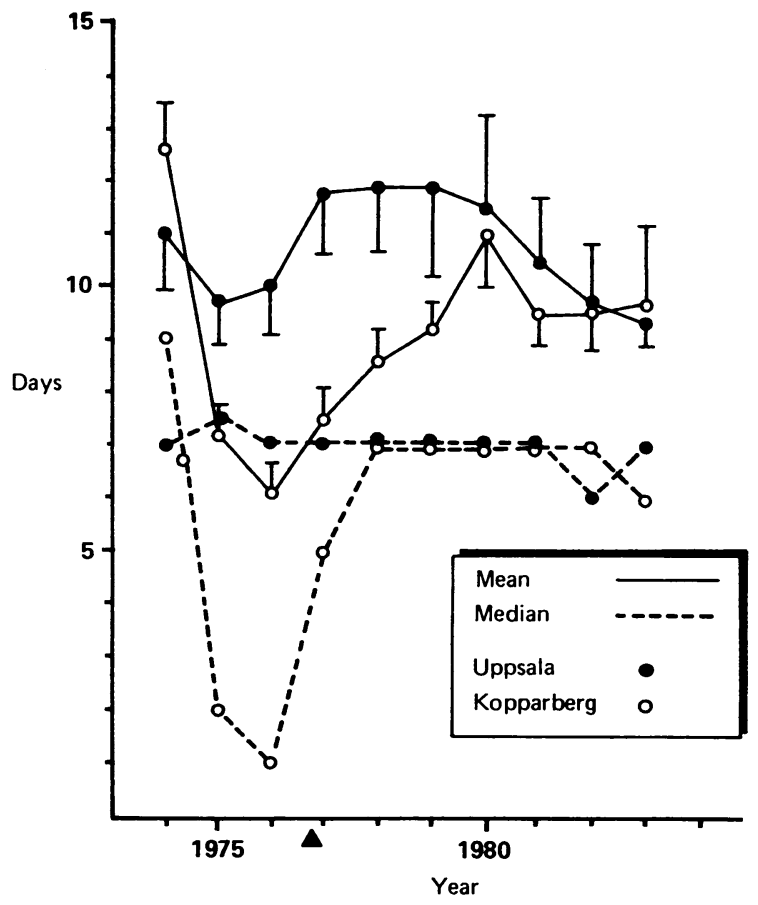

FIG 1-Annual median and mean (with SE) duration of hospital stay due to breast cancer in Uppsala and Kopparberg Counties 1972-82. $\boldsymbol{\Delta}=$ Beginning of screening programme.

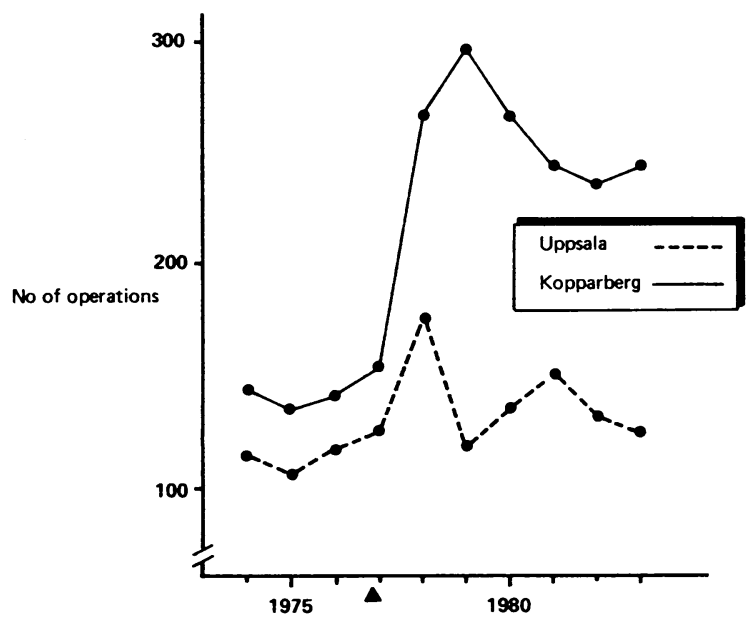

FIG 2-Annual numbers of inpatient operations for breast cancer in Uppsala and Kopparberg Counties.

October 1977. The mean values approached those for Uppsala during the first few years of the screening, and the median values were identical from 1978 onwards. These findings suggest that representative estimates of annual hospital stay before the mass screening programme, uninfluenced by adjuvant chemotherapy, can be obtained from mean values for Uppsala County.

The annual number of operations for malignant breast disease has slowly increased in Uppsala (fig 2) and in Sweden as a whole. ${ }^{14}$ In Kopparberg County the figures rose from an annual mean of 142 in the three years before the start of mammographic screening to a peak of 297 during that screening; they fell, however, during the last period of the study (see fig 2).

The annual increase in total annual hospital stay in surgical clinics due to breast cancer during the screening campaign (fig 3 ) corresponded with the increase in the number of operations. The higher baseline level in Kopparberg than in Uppsala, despite similarity in mean duration of stay and number of operations, is explained by the lack of an oncology clinic in Kopparberg. All women with recurrent breast cancer are treated at the oncological clinic in Uppsala County, whereas they are treated at the surgical clinics in Kopparberg. Hence hospital stay without operation is much more common in Kopparberg than in Uppsala. The pattern of a peak value during screening followed by a decline also appeared for total inpatient care per year. 


\section{CHANGES BY AGE GROUP}

Analysis of operations in Kopparberg County by age groups showed a high proportion of women aged 50-69 years, among whom the number of operations for breast cancer doubled during the initial round of screening (fig 4). Women aged 70 or more accounted for a smaller proportion of the total care, but there was an even greater increase in the number of operations in this group, despite a considerably lower participation rate than in the 50-69 year group (about 60\% v 92\%). Among women aged 40-49 screening had little or no effect on inpatient stay for breast cancer despite a high participation rate $(93 \%)$ and shorter interval between screening rounds.

\section{BENIGN BREAST LESIONS}

Fairly few operations were performed for non-malignant breast lesions (fig 5). The incidence was similar in both counties, as was the duration of

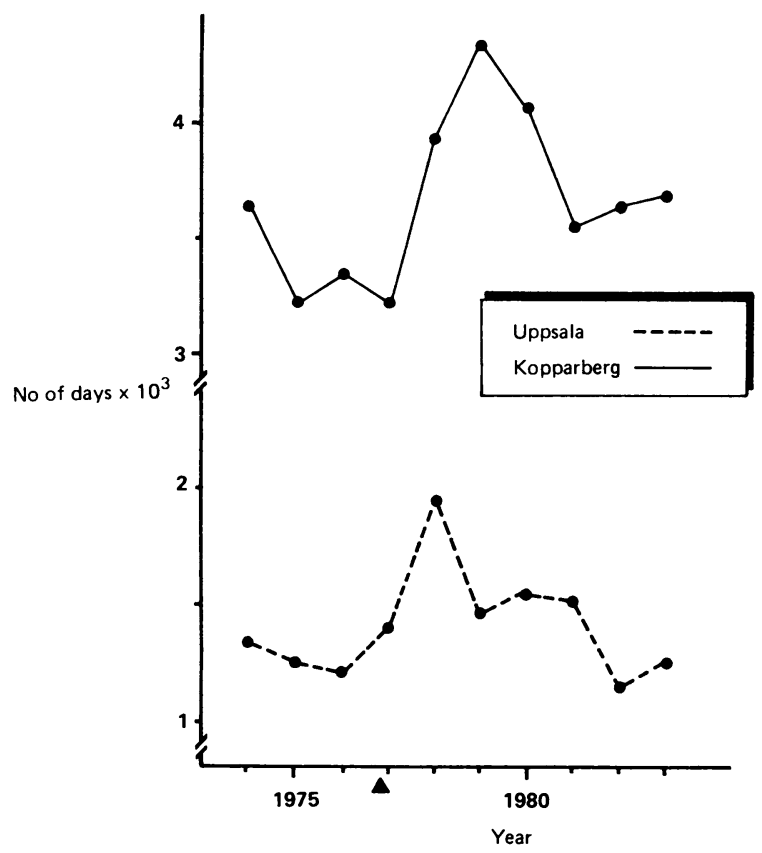

FIG 3-Total days of surgical care for breast cancer annually in Uppsala and Kopparberg Counties.

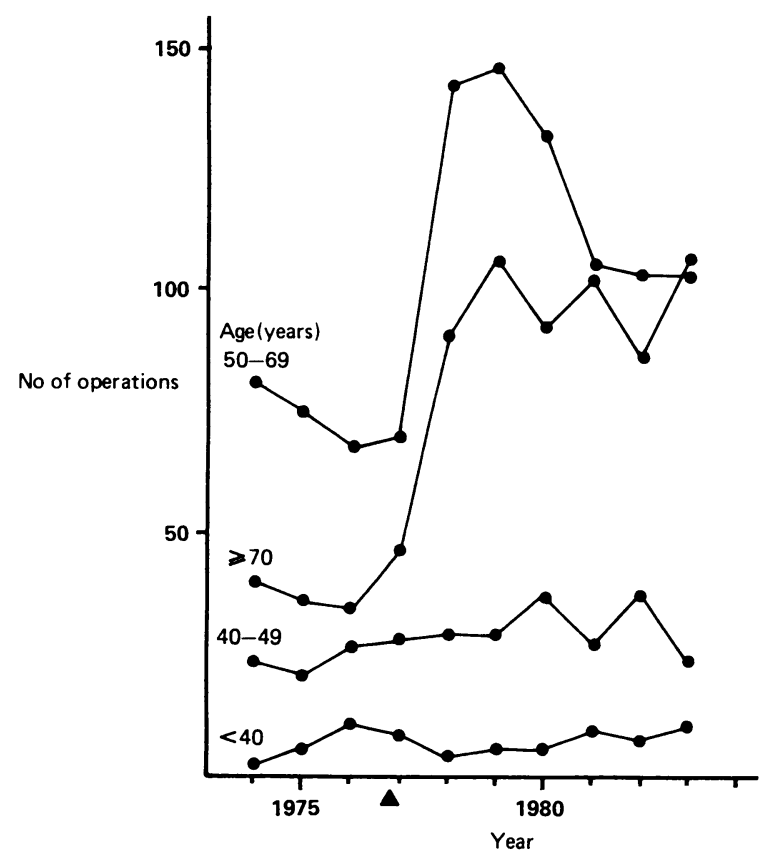

FIG 4-Age distribution of inpatient operations for breast cancer annually in Kopparberg County. hospital stay. During the early years of mammographic screening there was a slightly rising trend in Kopparberg County with an annual increase of about 20 operations for benign breast lesions, corresponding to about 100 days of inpatient care.

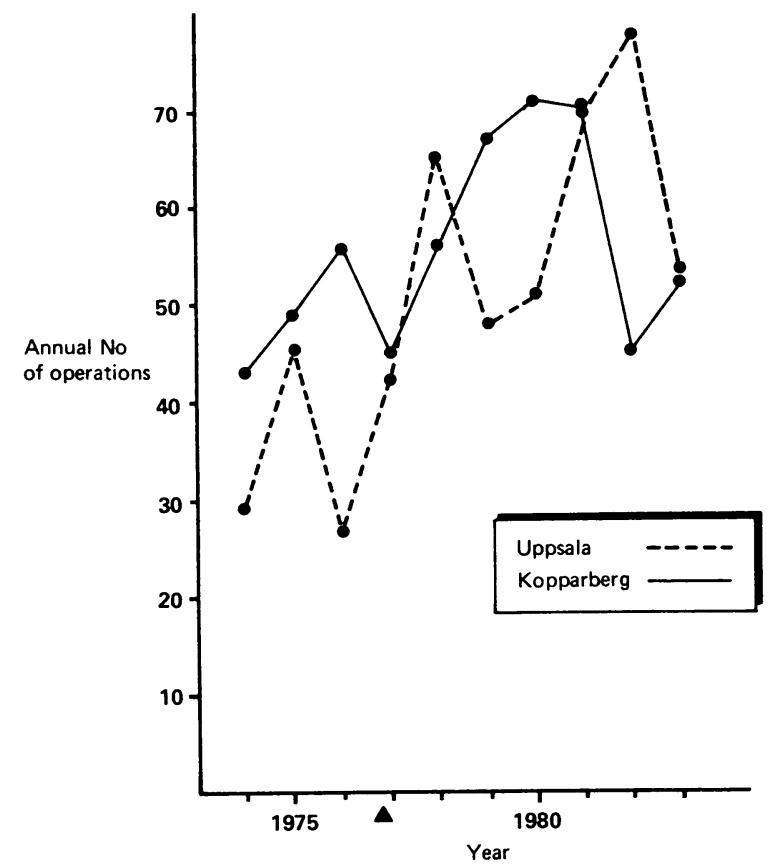

FIG 5-Annual numbers of inpatient operations for benign breast lesions in Uppsala and Kopparberg Counties.

\section{Discussion}

This analysis was based on a register of inpatients within a clearly defined population. The estimated number of cases not reported to the register was negligible. Strict histological and cytological criteria were applied for diagnosing breast cancer, and previous studies using the register have shown its precision in coding to be high. ${ }^{15}$

Only about two thirds of women aged above 40 were invited to the mammographic screening trial. Accordingly, increases in the numbers of operations, days in hospital, and so on, of about $50 \%$ more than reported here would follow a general screening programme with the same attendance rate.

After the prevalence screening was completed bed occupancy and the number of operations for breast cancer fell. This pattern reflects the falling incidence of cancer among screened women in the second and third rounds of screening towards levels in the control group (table). Thus we found that after an initial increase the demands on the health services levelled off as the numbers of cases detected by screening reached a steady state. The duration of the first round of screening is important for the shape of this peak - that is, if the time during which the population undergoes the first examination is short the workload will be very concentrated.

The age group covered also requires consideration. The time related changes in the demand for health care observed in other groups did not seem to apply to women aged $40-49$ years. Whether

Comparison of incidence of breast cancer (No of cases/1000 woman years) in study and control groups

\begin{tabular}{lccc}
\hline & \multicolumn{3}{c}{ Age range (years) } \\
\cline { 2 - 4 } & $40-49$ & $50-59$ & $60-69$ \\
\hline Control group & $1 \cdot 1$ & $2 \cdot 1$ & $2 \cdot 5$ \\
First screening & $2 \cdot 3$ & $4 \cdot 1$ & $8 \cdot 4$ \\
Second screening $^{\star}$ & $1 \cdot 6$ & $1 \cdot 4$ & $3 \cdot 0$ \\
Third screening $^{\star}$ & $1 \cdot 8$ & - & - \\
\hline
\end{tabular}

^ Cases of cancer between screenings included. 
this age group is invited to a screening programme or not-which is a matter of debate because the benefits of screening this group are still not clear ${ }^{8}$ - has little effect on demands for resources for surgical care. The high prevalence of cancer in women over 70, on the other hand, shows that this group should be accorded great importance in the planning of mammography screening. The study also indicated that screening programmes should be scheduled to invite women of mixed ages, not specific age groups.

Many mammographically diagnosed cancers (around 40\%) are detected at an occult stage and require preoperative radiographic localisation and perioperative mammographic examination of the specimen. The increase in the use of services will therefore be concentrated at hospitals where such resources are available.

The low incidence of operations for benign lesions of the breast and the insignificant effect of screening were unexpected, though they probably reflected the high specificity achieved in the trial $(98.9 \%) .{ }^{13}$ During the first round of screening two out of three women referred to surgery had breast cancer; the corresponding figures for the second round were three out of four. ${ }^{13}$ Low specificity, entailing referral to surgery for many women with benign and often occult lesions, can obviously increase the workload. The management of women requiring surgery for a mammographic lesion that is not unequivocally malignant is also important. Inpatient care may be advisable to achieve accurate preoperative diagnosis and radical removal of the lesion and for a better psychological care of the patient. Differing policies with regard to such cases will influence the demands made on resources for surgical inpatient care.

It is too early to make confident predictions about the long term consequences of mammographic screening for surgical inpatient services. The reduced absolute numbers of stage II and more advanced cancers found during screening ${ }^{16}$ and the increased diagnosis of non-palpable lesions should influence policies for diagnosis and treatment. An increasing number of women will be offered breast conserving treatment and fewer will undergo chemotherapy. Reduction in recurrence of and mortality from breast cancer should also have long term effects ${ }^{8}$ as the treatment of recurrent breast cancer is expensive and time consuming.

\section{References}

1 Logan WP. Cancer of the breast: no decline in mortality. WHO Chron 1975;29:462-71.

2 Rutquist LE. Increasing incidence and constant mortality rates of breast cancer: time trends in Stockholm County 1961-73. Breast Cancer Res Treat 1984;4:233-43.

Doll R, Peto $R$. The causes of cancer: quantitative estimates of avoidable risks of cancer in the United States today. 7 Natl Cancer Inst 1981;66:1191-308.

4 Anonymous. Review of mortality results in randomised trials in early breast cancer. Lancet 1984;ii: 1205.

5 Shapiro S. Ten to fourteen-year effect of screening on breast cancer mortality. $\mathcal{F}$ Natl Cancer Inst 1982;69:349-55.

6 Colette HJA, Day NE, Rombach JJ, deWaard F. Evaluation of screening for breast cancer in a non-randomized study (the DOM project) by means of a case-control study. Lancet 1984;i: 1224-6.

7 Verbeek ALM, Hendriks JHLC, Holland R, Mravunac M, Sturmans F, Day NE. Reduction of breast cancer mortality through mass screening with modern mammography. Lancet 1984 ; ; $1222-4$.

8 Tabar L, Fagerberg CJG, Gad A, et al. Reduction in mortality from breast cancer after mass screening with mammography. Lancet 1985;i:829-32.

9 Swedish Board of Health and Welfare. In-patient statistics from hospitals for physical diseases in the Uppsala region. Stockholm: Swedish Board of Health and Welfare, 1969. (Patient statistics No 1.)

10 Swedish Board of Health and Welfare. The users of hospital care. Stockholm: Swedish Board of Health and Welfare, 1972. (Patient statistics No 11.)

11 Bonadonna G, Brusamolino E, Valagussa $P$, et al. Combination chemotherapy as an adjuvant treatment in operable breast cancer. N Englf Med 1976;297:405-10.

12 Tabar L, Gad A. Screening for breast cancer: the Swedish trial. Radiology 1981;138:219-22.

13 Tabar L, Gad A, Akerlund E, Holmberg L. Screening for breast cancer in Sweden. In: Feig S, McLelland R, eds. Breast carcinoma: current diagnosis and treatment. New York: Masson Pupl USA Inc, 1983.

14 National Board of Health and Welfare. Cancer incidence in Sweden 1980. Stockholm: National Board of Health and Welfare, 1984.

15 Adami HO, Meirik O, Gustavsson S, Nyrén O, Krusemo U-B. Colorectal cancer after cholecystectomy: absence of risk increase within 11-14 years. Gastroenterology 1983;85:859-65.

16 Tabar L, Gad A, Holmberg L, Ljungquist U. Significant reduction in advanced breast cancer. Results of the first seven years of mammography screening in Kopparberg, Sweden. Diagnostic Imaging in Clinical Medicine 1985;54:158-64.

(Accepted 3 fuly 1986)

\section{Abstract}

Serum samples from 6015 African subjects without symptoms of the acquired immune deficiency syndrome (AIDS) or contact with the disease were examined for antibodies to the human immunodeficiency virus by a combination of an enzyme linked immunosorbent assay and radioimmunoprecipitation (2567 samples) or by immunofluorescence (3448 samples). Serum samples had been collected between 1976 and 1984 in Senegal

\footnotetext{
Deutsches Primatenzentrum, D-3400 Göttingen, Federal Republic of Germany

I WENDLER, PHD, research assistant

J SCHNEIDER, PHD, research assistant

G HUNSMANN, MD, head of virology and immunology section, professor of medicine
}

Bernhard Nocht Institut, D-2000 Hamburg 4, Federal Republic of Germany

B GRAS, medical student, department of virology

H SCHMITZ, MD, head of department of virology, professor of medicine

Tropical Diseases Research Centre, Ndola, Zambia

A F FLEMING, MD, deputy director of research, professor of medicine

Correspondence and requests for reprints to: Professor Dr Gerhard Hunsmann, Department of Virology and Immunology, Deutsches Primatenzentrum, Kellnerweg 4, D-3400 Göttingen, Federal Republic of Germany. (n=789), Liberia (935), Ivory Coast (1195), Burkina Faso (299), Nigeria (536), Gabon (1649), Zaire (15), Uganda (164), and Kenya (433). Only four samples contained antibodies. Three of these were from attenders at the Lambarene clinic in Gabon and one from a villager in Senegal. By contrast, two out of six AIDS suspects from Guinea-Bissau, all 13 patients with AIDS from Kinshasa (Zaire), and two out of three of their contacts were seropositive, all these specimens having been collected in 1985 .

These data show that fewer than one in a 1000 subjects were seropositive for AIDS at the time of sampling before 1985 and do not support the hypothesis of the disease originating in Africa.

\section{Introduction}

The acquired immune deficiency syndrome (AIDS) was diagnosed in patients from equatorial Africa from about 1981 at roughly the same time as the epidemic was recognised in North America and Europe, and AIDS is now widespread throughout central and eastern Africa. ${ }^{1.5}$ Transmission in tropical Africa differs from that in North America and Europe, being primarily by heterosexual contact; risk groups have been identified as urban dwelling, wealthy, mobile, and promiscuous heterosexual men, their wives and children, and women prostitutes. ${ }^{5-12}$ Only in South Africa is transmission more common among homosexual men than in other groups. ${ }^{13}$ 